\title{
Extended SEIR Model for Health Policies Assessment Against the COVID-19 Pandemic: the Case of Argentina
}

\section{Fernando A. Inthamoussou ${ }^{1}$ (D) . Fernando Valenciaga ${ }^{1}$ • Sebastián Núñez ${ }^{1}$. Fabricio Garelli ${ }^{1}$}

Received: 1 April 2021 / Revised: 11 August 2021 / Accepted: 2 November 2021 /

Published online: 7 December 2021

(C) The Author(s), under exclusive licence to Springer Nature Switzerland AG 2021

\begin{abstract}
This work presents an extended and age-band compartmentalised SEIR model that allows describing the spread evolution of SARS-CoV-2 and evaluating the effect of different detection rates, vaccination strategies or immunity periods. The model splits up the population into fifteen age groups of 5 years each, linked through a statistical interaction matrix that includes seventeen health states within each age group. An age-dependent transmission rate takes into account infectious between the groups as well the effect of interventions such as quarantines and mobility restrictions. Further, the proposal includes a nonlinear switched controller for model tuning purposes guarantying a simple and fast adjusting process. To illustrate the model potentials, the particular case of COVID-19 evolution in Argentina is analysed by simulation of three scenarios: (i) different detection levels combined with mobility restrictions, (ii) vaccination campaigns with re-opening of activities and (iii) vaccination campaigns with possible reinfections. The results exhibit how the model can aid the authorities in the decision making process.
\end{abstract}

Keywords COVID-19 · SARS-CoV-2 · Extended SEIR Model · Vaccination · Immunity loss

This article belongs to the Topical Collection: Special Collection on COVID-19 Guest Editors: Christopher C. Yang, Julio Cesar Facelli, David Buckeridge, Fei Wang

Fernando A. Inthamoussou

intha@ing.unlp.edu.ar

1 Grupo de Control Aplicado (GCA) LEICI. Facultad de Ingeniería, UNLP - CONICET, CC 91, CP 1900, La Plata, Buenos Aires, Argentina 


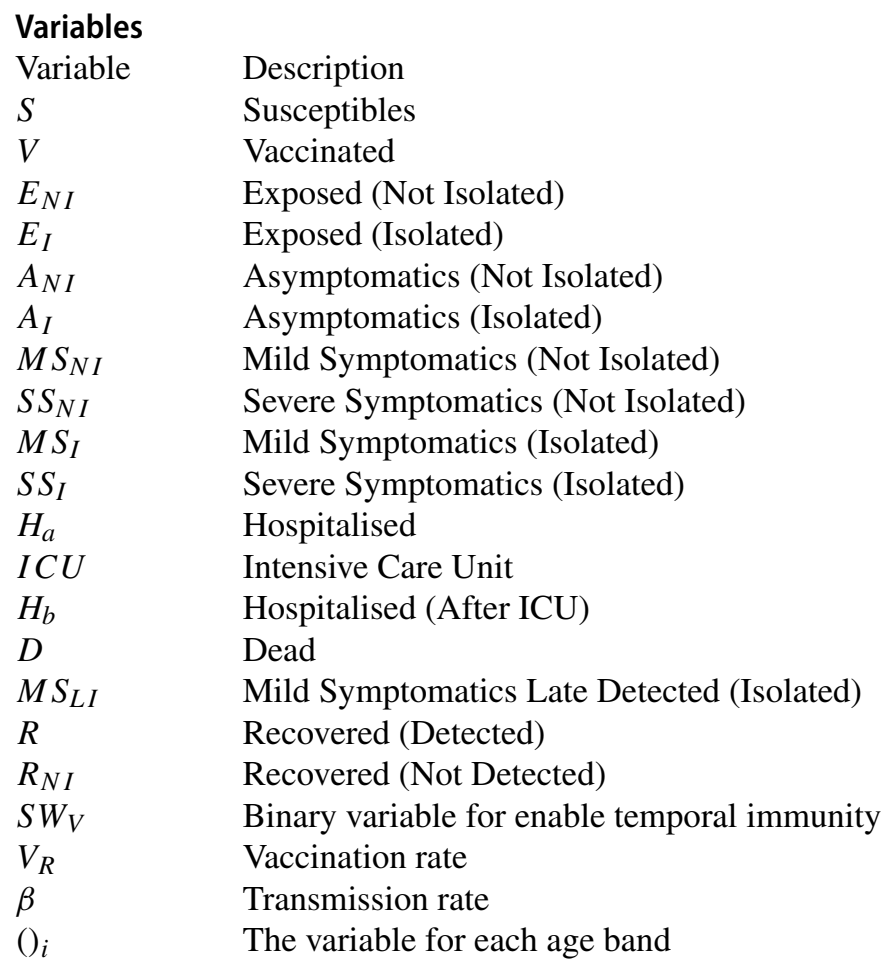

\section{Abbreviations}

Abbreviation

Description

ICU

Intensive care units

NPIs

Non pharmaceutical interventions

SARS-CoV-2 Severe acute respiratory syndrome coronavirus 2

SEIR Susceptible - Exposed - Infected - Recovered

SIR

Susceptible - Infected - Recovered

SIS

Susceptible - Infected - Susceptible

\section{Parameters}

Variable

$T_{e}$

$T_{a}$

$T_{t}$

$T_{l 1}$

$T_{l 2}$

$T_{r 1}$

$T_{r 2}$

$T_{d}$

$T_{h}$
Description

Average time on $E_{I}$ and $E_{N I}$

Average time on $A_{I}$ and $A_{N I}$

Average time from $H_{a}$ to $I C U$

Average time on $M S_{N I}$ and $M S_{I}$

Average time on $M S_{L I}$

Average time from $H_{a}$ to $R$

Average time on $H_{b}$

Average time from $I C U$ to $D$

Average time from $I C U$ to $H_{b}$ 


$\begin{array}{ll}T_{r} & \text { Average time on } R \text { and } R_{N I} \\ T_{i m} & \text { Average time on } V \\ \sigma_{s} & \text { Probability of severe symptoms } \\ \mu & \text { Probability of } H_{a} \text { to } I C U \\ \rho & \text { Probability of } I C U \text { to } D \\ \lambda & \text { SARS-CoV-2 free transmission coefficient } \\ \delta & \text { Detection probability } \\ \epsilon & \text { Probability of late detection } \\ N & \text { Total population under study } \\ r_{a} & \text { Transmission capability of non-hospitalised isolated infectious } \\ r_{h} & \text { Transmission capability of hospitalised isolated infectious } \\ r_{c} & \text { Transmission capability of non-isolated infectious } \\ ()_{i} & \text { The parameter for each age band }\end{array}$

\section{Introduction}

The present SARS-CoV-2 epidemic has triggered lots of enthusiastic research in different scientific fields with the objective of understanding its behaviour and finding tools to mitigate its effects. Non pharmaceutical interventions (NPIs) like quarantines, social distancing, school/university attendance suspension, massive events prohibition, the use of masks and exhaustive testing have been up to the moment the only effective measures found to retain the virus spread [1]. These interventions only aim to diminish the virus dissemination speed to avoid the health service saturation, especially in the case of intensive care units (ICU) with ventilators. At the time of writing this paper some countries were starting to vaccinate a reduced number of people, using vaccines approved for emergency use. However, due to the reduced production numbers and logistics problems, NPIs are still the only effective measures available for most countries administrations.

The delayed effect that interventions have on the SARS-CoV-2 dissemination makes difficult to impose long social restrictions affecting entire populations and economy. In this uncertain context, and taking in mind the close vaccines availability, it is important to have systematic and reliable tools that allow predicting the relative efficiency of different pharmaceutical and non-pharmaceutical interventions, their potential combinations, length, intermittence, depth, etc. helping to take appropriate decisions. Then, it becomes relevant to develop and tune new epidemic dynamic models able to describe the behaviour of new infectious illnesses [2-5].

The disease transmission process can be described by mass-action kinetics using differential equations resulting in analytic expressions [6]. In this sort of models, the community members are aggregated into a few compartments identified with the different states of the disease. These models are characterised by the rates of transition among compartments. Then, the model complexity is determined by the number of compartments and pathways required to describe the disease dynamics with sufficient accuracy [7, 8]. An homogeneous population is implicitly assumed in the above definitions. Age, geographic, social-economic and activity heterogeneity 
can be included into the model yielding more compartments and a contact matrix interconnecting the groups [9-11].

Simpler and well-known compartmental models are the Susceptible - Infected (SI), the Susceptible - Infected - Susceptible (SIS), the Susceptible - Infected - Recovered (SIR) and the Susceptible - Exposed - Infected - Recovered (SEIR). All of them consider a reduced number of health status states setting up the basis for more exhaustive dynamic models $[16,17]$. To deeply evaluate the health system occupancy level, consider the effects of undetected cases, assess the effectiveness of social restrictions, and look for optimal vaccination strategies among other alternatives, dynamic models that consider a greater number of intermediate health status are necessary. It is also essential to classify the considered population into several age bands and to incorporate their social interconnections. Besides, these models must present enough inputs, coefficients or parameters to describe possible pharmaceutical and non-pharmaceutical interventions $[12,13,18]$. Interesting reviews about these topics can be found in $[14,15]$.

In the described context, the present work introduces an extended compartmental SEIR dynamic model that allows predicting the disease evolution, the detection campaigns and isolation policies effectiveness (jointly applied or not), the effects of different vaccination strategies with or without immunity loss, and the local consequences produced by imported cases. To this end, the considered population is divided in fifteen age bands of 5-year width, each one of them described by a seventeen health status dynamic model. All of these models are interconnected through a function that interprets the epidemic transmission channels, including several parameters and coefficients that allow representing different intervention policies (social distancing, general quarantines or mobility restrictions, use of masks, etc.). This function is modulated by a matrix that considers the risky contacts (in terms of virus transmission) among people from different age bands. This matrix, usually called risk contact matrix, characterises in some way the social behaviour of a given population. The proposed segmented model permits the user to include different parameters values for each age band, thus allowing a better characterisation of their particularities and dynamic behaviours. Differing from other epidemiological models, the proposed one here can be used to evaluate different vaccination campaigns, the potential loss of immunity and considers the imported cases. Also, the contagion rate function uses the risk contact matrix only for the non-isolated cases and considers different control coefficients for the hospitalised, isolated and not isolated cases. Further, the proposal includes a switched controller for model tuning purposes. This controller is only used for adjusting unknown parameters due to the NPI policies. The inclusion of this controller simplifies adjusting the model against actual data. The proposed model was tuned to the Argentinian case using the official statistics properly processed. Three simulation scenarios (including different testing levels and vaccination strategies, the opening of some activities and possible immunity loss) illustrate the model capacity and potential as a decision support framework for this and future pandemics. 


\section{Model}

The proposed epidemiological dynamic model is built around a classic SEIR topology properly modified to take into account an extended set of intermediate health status compartments, the effect of imported cases, detection campaigns, isolation and mobility restrictions interventions, the risky contacts among individuals, the mediumterm consequences of partial vaccination schemes and lost of immunity. To represent these effects the whole model is developed in fifteen 5-year width layers (or age bands) linked through a transmission rate function which depends on an inter-age risk contacts matrix, typical of each country (cultural effects).

A schematic representation of each one of these model layers is depicted in Fig. 1. This plot shows a compartmental structure with seventeen health status blocks. They are mainly organised in two branches that partially converge at the right side of the figure where several blocks related to health conditions that require hospitalisation take place.

Specifically, the upper orange branch represents the health status sequence corresponding to infected people early detected and isolated. The bottom branch in blue considers the health status progress of infected people not early detected. Starting from a healthy population of $N$ individuals without specific antibodies against SARS-CoV-2, the upper branch model describes the health status sequence followed by infected individuals early detected and isolated through testing campaigns. After being infected with a transmission rate $\beta$ and detected with a probability $\delta$, these exposed isolated individuals $\left(E_{I}\right)$ become contagious after $T_{e}$ days without presenting any symptoms. This asymptomatic contagious illness stage $\left(A_{I}\right)$ lasts $T_{a}$ days on average. After this period, an age-dependent portion $\sigma_{s}$ of these individuals start to exhibit severe symptoms $\left(S S_{I}\right)$ and require hospitalisation. The rest of them only experiment mild symptoms $\left(M S_{I}\right)$ being capable to go through the illness process without any serious trouble, recovering their healthy status $(R)$ after $T_{l 1}$ days on average. On the other hand, non-detected exposed individuals $\left(E_{N I}\right)$ develop an identical health status evolution characterised by the same illness parameters $\left(T_{e}, T_{a}\right.$ and

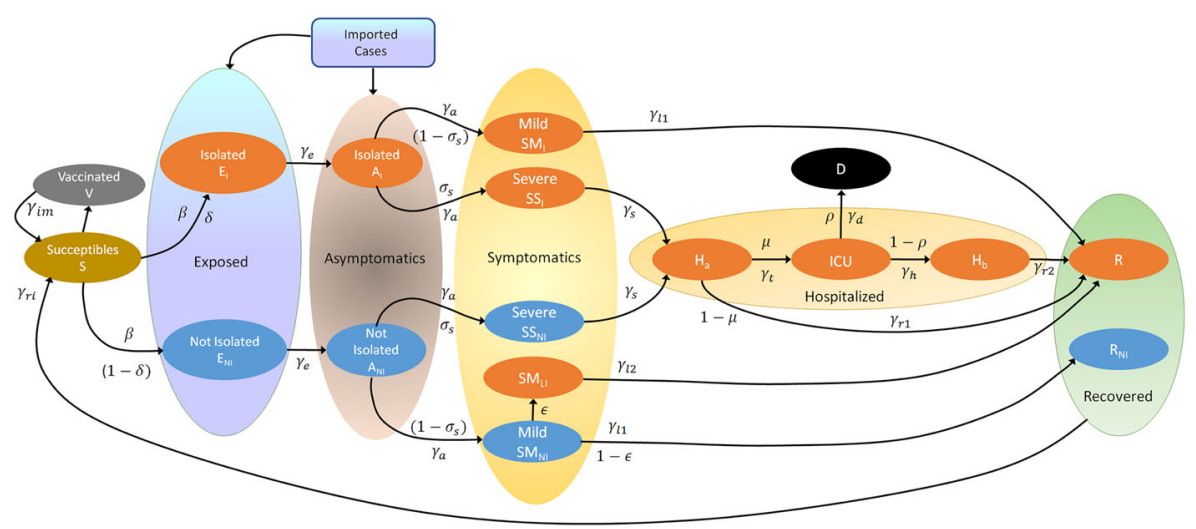

Fig. 1 Schematic structure of the compartmental COVID-19 model 
$\left.\sigma_{s}\right)$. However, unlike the previous case, the model considers that a portion $\epsilon$ of nonisolated individuals presenting mild symptoms can be late detected $\left(M S_{L I}\right)$ by testing campaigns or medical consultation, and properly isolated during a safe period of $T_{l 2}$ days. The rest of them $\left(M S_{N I}\right)$, like in the upper line, can be considered completely recovered $\left(R_{N I}\right)$ after a period of $T_{l 1}$ days on average. All individuals presenting severe symptoms, being previously isolated or not, are finally admitted in health institutions for control and care. Typically, after $T_{t}$ days on average, an age-dependent portion $\mu$ of these patients develop breathing complications and must be moved to ICU equipped with ventilators. The remaining patients run through the illness process with common cares and, after an average period of $T_{r 1}$ days, they are discharged from hospital and considered recovered $(R)$. Regretfully, an age-dependent portion $\rho$ of those who were admitted in ICU will finally die after an average period of $T_{d}$ days. The others will stay there for average period of $T_{h}$ days, after which they will return to common care units $\left(H_{b}\right)$ to complete recovery treatments before being discharged from hospital. This last process will demand a period of $T_{r 2}$ days on average. As it can be seen in Fig. 1, the compartment of recovered people is composed by two sub-blocks, $R$ and $R_{N I}$, allowing differentiating between individuals who have been detected and isolated in any moment of the illness period or not. Anyway, all recovered patients are supposed to acquire temporal specific antibodies against SARS-CoV-2. Despite that it is even uncertain nowadays, the possible specific medium-life of immunity is modelled through a period of $T_{r i}$ days, after which these individuals would return to the susceptible compartment and could be reinfected. This path can be enabled in simulations through the binary parameter $S W_{R}$, where $S W_{R}=0$ means that immunity is permanent. Vaccination campaigns are also considered through the $V$ state which is feed by the vaccination rate $(V R)$. A possible $T_{i m}$ days average period of temporal immunity can be enabled through the binary parameter $S W_{V}$. Imported cases $(I C)$ can be considered through direct input feed paths to the exposed, symptomatic and asymptomatic compartments, on the detected or non-detected branch either. For a matter of simplicity and available data, the imported cases enters at the isolated asymptomatic compartment.

At this point, it is important to emphasise that the explained model assumes the following simplifications: severe cases are treated at hospital while patients developing smooth symptoms stay at home; no previous co-morbidity nor sex distinction is taken into account; ICU patients come from previous hospital stages only and finally, each age band has a fixed initial population, i.e. births, mortality by other causes and moving between age bands are not considered. This last assumption is fairly true for short/medium term simulations. Following the previous description and taking into account the nomenclature of Fig. 1, the dynamic expressions corresponding to the states of each age band $(i=1 \ldots 15)$ can be analytically written as shown in Table 1.

The sub-index $i$ is also used to denote the age-dependent parameters typical of each age band (see Table 1). Besides, it should be noted that $\gamma_{x}=1 / T_{x}$.

The proposed age-dependent transmission rate allows considering different kinds of epidemic qualitative control measures including pharmaceutical treatments, NPIs 
Table 1 System dynamics

$$
\begin{aligned}
& \dot{S}_{i}=-\beta_{i} S_{i}+\gamma_{i m} V S W_{V}+\gamma_{r i}\left(R+R_{N I}\right) S W_{R}-V R \\
& \dot{V}=V R-\gamma_{i m} V S W_{V} \\
& \dot{E}_{N I_{i}}=(1-\delta) \beta_{i} S_{i}-\gamma_{e} E_{N I_{i}} \\
& \dot{E}_{I_{i}}=\delta \beta_{i} S_{i}-\gamma_{e} E_{I_{i}} \\
& \dot{A}_{N I_{i}}=\gamma_{e} E_{N I_{i}}-\gamma_{a} A_{N I_{i}} \\
& \dot{A}_{I_{i}}=\gamma_{e} E_{I_{i}}-\gamma_{a} A_{I_{i}}+I C \\
& \dot{M} S_{N I_{i}}=\left(1-\sigma_{s_{i}}\right) \gamma_{a} A_{N I_{i}}-(1-\epsilon) \gamma_{L 1} M S_{N I_{i}}-\epsilon M S_{N I_{i}} \\
& \dot{S} S_{N I_{i}}=\sigma_{s_{i}} \gamma_{a} A_{N I_{i}}-\gamma_{s} S S_{N I_{i}} \\
& \dot{M} S_{I_{i}}=\left(1-\sigma_{s_{i}}\right) \gamma_{a} A_{I_{i}}-\gamma_{L 1} M S_{I_{i}} \\
& \dot{S} S_{I_{i}}=\sigma_{s_{i}} \gamma_{a} A_{I_{i}}-\gamma_{s} S S_{I_{i}} \\
& \dot{H}_{a_{i}}=\gamma_{s}\left(S S_{N I_{i}}+S S_{I_{i}}\right)-\mu_{i} \gamma_{t} H_{a_{i}}-\left(1-\mu_{i}\right) \gamma_{r 1} H_{a_{i}} \\
& I \dot{C} U_{i}=\mu_{i} \gamma_{t} H_{a_{i}}-\left(1-\rho_{i}\right) \gamma_{h} I C U_{i}-\rho_{i} \gamma_{d_{i}} I C U_{i} \\
& \dot{H}_{b_{i}}=\left(1-\rho_{i}\right) \gamma_{h} I C U_{i}-\gamma_{r 2} H_{b_{i}} \\
& \dot{D}=\rho_{i} \gamma_{d} I C U_{i} \\
& \dot{M} S_{L I_{i}}=\epsilon M S_{N I_{i}}-\gamma_{L 2} M S_{L I_{i}} \\
& \dot{R}=\gamma_{l 1} M S_{I_{i}}+\gamma_{r 2} H_{b_{i}}+\left(1-\mu_{i}\right) \gamma_{r 1} H_{a_{i}}+\gamma_{l 2} M S_{L I_{i}} \\
& \dot{R}{ }_{N I}=(1-\epsilon) \gamma_{l 1} M S_{N I_{i}}
\end{aligned}
$$

(quarantines, isolation, social distance, etc.) and stepped/partial vaccination campaigns. In this way, the proposed dynamic model constitutes a worthy tool to appropriately evaluate different schemes of epidemic control measures and vaccination. Thus, it can be used as a decision support framework to reduce the fatal cases and the epidemic length. Specifically, this age-dependent transmission rate $\beta_{i}$ can be written as shown in Table 2.

This expression presents several terms, tuning coefficients and a general constant parameter $\lambda$ that affects the whole expression because it represents the SARS-CoV-2 characteristic transmission coefficient. The first term of the equation in Table 2 takes into account the total non-detected infected people able to freely spread the virus into the community. This transmission effect is not uniform over all the age bands. It is weighted considering a risk contact matrix of elements $k_{i j}$ that compute those risk contacts among individuals of equal or different age bands where the SARSCoV-2 can be transmitted [19, 20]. Figure 2 graphically shows the aspect of the risk contact matrix. It is interesting to note that this matrix assumes different shapes for each society or culture. Specific activities suspension like school, university, etc. are considered through modulation of the risk contact matrix on the block corresponding to the particular age bands involved. The effect of quarantines, social distance measures or partial mobility restrictions is uniformly modulated by the coefficient $r_{c}$. The second term of the equation in Table 2 involves the total amount of non-hospitalised isolated infectious people. Because of their condition, these patients

Table 2 Contagion rate function

$$
\beta_{i}=\lambda\left(r_{c} \Sigma_{j=1}^{15} k_{i j} \frac{\left(A_{N I_{j}}+M S_{N I_{j}}+S S_{N I_{j}}\right)}{N_{j}}+r_{a} \Sigma_{j=1}^{15} \frac{\left(M S_{L I_{j}}+A_{I_{j}}+M S_{I_{j}}+S S_{I_{j}}\right)}{N_{j}}+r_{h_{i}} \Sigma_{j=1}^{15} \frac{\left(H_{a_{j}}+I C U_{j}\right)}{N_{j}}\right)
$$




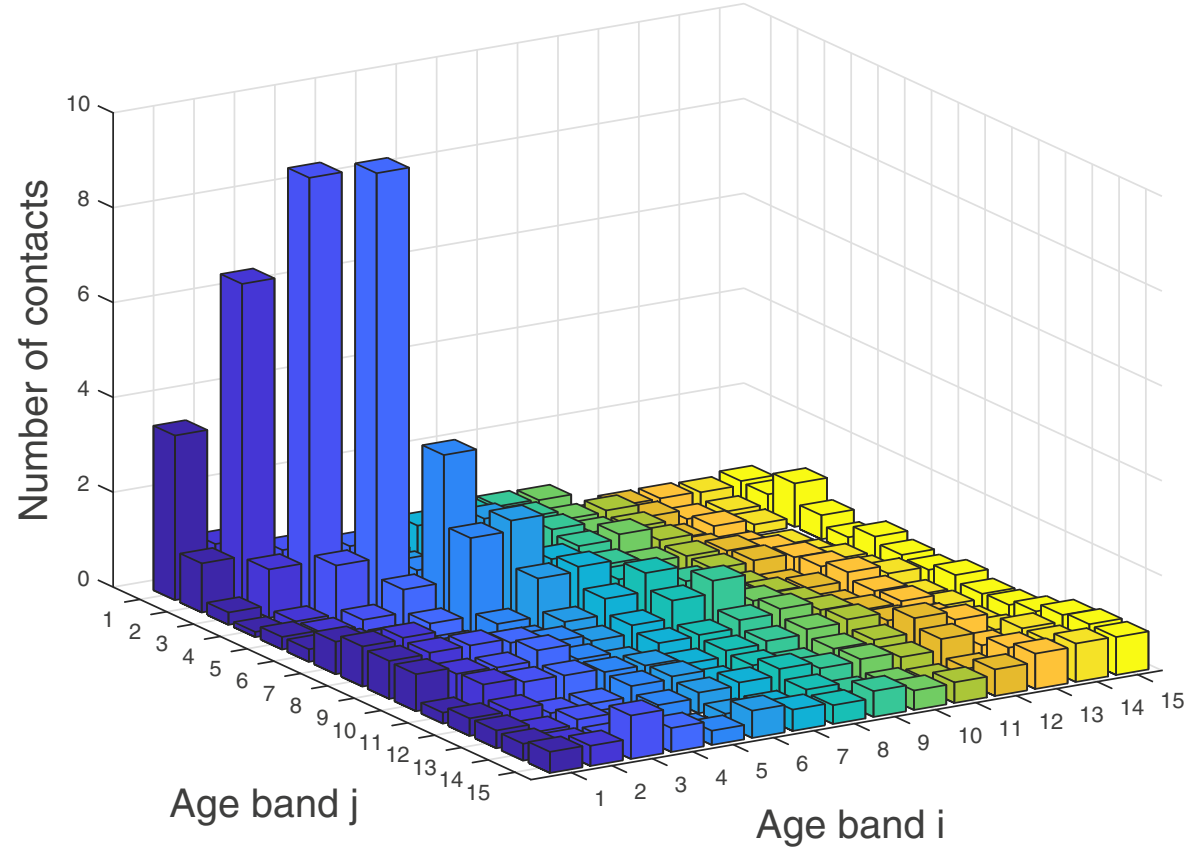

Fig. 2 Risk contacts matrix (5-year width bands)

do not transmit the virus freely. Indeed, they could only transmit the infection to housemates, ideally. However, to simplify the model construction a reduced and uniform disease distribution effect is considered. Then coefficient $r_{a}$ is used to reflect the transmission capability of this group of people. Finally, the third term of the equation in Table 2 considers the transmission capability corresponding to hospitalised people over each one of the fifteen age bands. Given that these patients can only infect hospital workers, this effect is taken into account through a vector of elements $r_{h_{i}}$. It is straightforward to see that some of these vector elements are zero (the first and last four age bands).

Partial or stepped vaccination campaigns can be considered through a double action on the described model. Firstly, the equivalent percentage of artificially immunised people must be removed from the susceptible compartment of the corresponding age band. This group stays in a separate compartment (see Fig. 1) during the immunisation period before returning to the compartment of susceptible people. On the other hand, the risk contact matrix column corresponding to the vaccinated age band should be affected by a coefficient equal to the immunised people percentage to take into account close contacts between susceptible and immunised persons.

\section{Model Adjustment: the Argentinian Case}

The model presented in the previous section was adjusted to represent the pandemic evolution in Argentina by using the National Health Ministry database adequately 
Table 3 Transition speeds

\begin{tabular}{lll}
\hline Transition & Parameter & Value \\
\hline$E \rightarrow A_{x}$ & $\gamma_{e}=1 / T_{e}$ & $1 / 5.1$ \\
$A_{x} \rightarrow S_{x}$ & $\gamma_{a}=1 / T_{a}$ & $1 / 2$ \\
$S S_{x} \rightarrow H_{a}$ & $\gamma_{s}=1 / T_{S}$ & $1 / 10$ \\
$M S_{x} \rightarrow R$ & $\gamma_{l 1}=1 / T_{l 1}$ & $1 / 11$ \\
$M S_{l a} \rightarrow R$ & $\gamma_{l 2}=1 / T_{l 2}$ & $1 / 11$ \\
$H_{a} \rightarrow R$ & $\gamma_{r 1}=1 / T_{r 1}$ & $1 / 10$ \\
$H_{a} \rightarrow I C U$ & $\gamma_{t}=1 / T_{t}$ & $1 / 3$ \\
$I C U \rightarrow H_{b}$ & $\gamma_{h}=1 / T_{h}$ & $1 / 19$ \\
$I C U \rightarrow D$ & $\gamma_{d}=1 / T_{d}$ & $1 / 14$ \\
$H_{b} \rightarrow R$ & $\gamma_{r 2}=1 / T_{r 2}$ & $1 / 10$ \\
$R \rightarrow S$ & $\gamma_{r i}=1 / T_{r i}$ & $1 / 180$ \\
$V \rightarrow S$ & $\gamma_{i m}=1 / T_{i m}$ & $1 / 360$ \\
\hline
\end{tabular}

processed [21]. Although this public register presents many data fields, it does not allow to determine all the model parameters. To overcome this gap some of them were then taken from the specialised literature. Table 3 shows the parameters obtained from the literature [22-24]. Tables 4 and 5 show the parameters obtained from National Health Ministry databases. In Table $4, \sigma_{s}, \mu$ and $\rho$ are shared by more than one age band due to their similarities.

As it was established in the equation of $\beta_{i}$ (Table 2) the transmission rate is highly dependent on the daily risk contacts matrix [19, 20]. This matrix, obtained from [20], counts and classifies by age band all those interpersonal contacts considered as a positive virus transmission meet in a day. Figure 2 shows a 3D bars representation of the symmetrised risk contact matrix used for simulations. It is interesting to note in this plot the high number of risky contacts among low-age bands clearly determined by school attendance. This matrix is helpful to recreate partial restrictions or particular

Table 4 Transition probabilities

\begin{tabular}{llll}
\hline Age band (years) & $\begin{array}{l}A_{x} \rightarrow S S_{x} \\
\sigma_{s}\end{array}$ & $\begin{array}{l}H_{a} \rightarrow I C U \\
\mu\end{array}$ & $\begin{array}{l}I C U \rightarrow D \\
\rho\end{array}$ \\
\hline $\mathbf{0 - 1 9}$ & 0.0489 & 0.0192 & 0.3193 \\
$\mathbf{2 0 - 2 9}$ & 0.0287 & 0.0138 & 0.5167 \\
$\mathbf{3 0 - 3 9}$ & 0.0349 & 0.0205 & 0.5657 \\
$\mathbf{4 0 - 4 4}$ & 0.0414 & 0.0412 & 0.6579 \\
$\mathbf{4 5 - 4 9}$ & 0.0512 & 0.0634 & 0.6938 \\
$\mathbf{5 0 - 5 4}$ & 0.0632 & 0.0873 & 0.7046 \\
$\mathbf{5 5 - 5 9}$ & 0.0810 & 0.1115 & 0.7563 \\
$\mathbf{6 0 - 6 4}$ & 0.1146 & 0.1479 & 0.8158 \\
$\mathbf{6 5 - 6 9}$ & 0.1510 & 0.1581 & 0.8541 \\
$\mathbf{+ 7 0}$ & 0.2508 & 0.1032 & 0.8847 \\
\hline
\end{tabular}


Table 5 Argentinian population by ages (Population Census 2010)

\begin{tabular}{llll}
\hline $\begin{array}{l}\text { Age band } \\
\text { (years) }\end{array}$ & Population & $\begin{array}{l}\text { Age band } \\
\text { (years) }\end{array}$ & Population \\
\hline $0-4\left(N_{1}\right)$ & $3,306,953$ & $40-44\left(N_{9}\right)$ & $2,176,030$ \\
$5-9\left(N_{2}\right)$ & $3,327,517$ & $45-49\left(N_{10}\right)$ & $2,074,112$ \\
$10-14\left(N_{3}\right)$ & $3,447,535$ & $50-54\left(N_{11}\right)$ & $1,932,694$ \\
$15-19\left(N_{4}\right)$ & $3,456,528$ & $55-59\left(N_{12}\right)$ & $1,761,202$ \\
$20-24\left(N_{5}\right)$ & $3,154,614$ & $60-64\left(N_{13}\right)$ & $1,491,271$ \\
$25-29\left(N_{6}\right)$ & $2,965,049$ & $65-69\left(N_{14}\right)$ & $1,188,958$ \\
$30-34\left(N_{7}\right)$ & $2,952,844$ & $+70\left(N_{15}\right)$ & $2,811,587$ \\
$35-39\left(N_{8}\right)$ & $2,536,248$ & Total & $38,583,142$ \\
\hline
\end{tabular}

activities cancellation during simulations. For example, school attendance suspension can be emulated by reducing the matrix values corresponding to age bands involved in these activities. In the same way, elderly people staying at home can be represented by affecting the last three rows and columns of the risk contacts matrix by an appropriate reduction factor.

At this point, to completely characterise the model, it remains to determine the coefficients $r_{a}, r_{h}$ and $r_{c}$ involved in $\beta_{i}$ (Table 2) and the parameters $\delta, \epsilon$ and $\lambda$. As it was previously specified, $r_{a}$ is a factor that modulates the transmission rate of infected isolated individuals over the rest of the population. Considering the effectiveness of isolation in transmission reduction, this constant was assumed as $r_{a}=0.05$ for all the age bands. In the case of the vector $r_{h}$, each one of its fifteen elements $\left(r_{h i}\right)$ modulates the transmission effect of the hospitalised patients (patients in $H_{a}$ and $I C U$ ) over the considered age band. Supposing that the hospital's staff only involves middle-age workers, the first five and the last three elements of $r_{h}$ were considered zero. Assuming that these institutions preserve a high prophylaxis level, the remaining elements of $r_{h}$ were fixed in 0.05. Finally, coefficient $r_{c}$ represents a transmission rate modulation factor that mainly considers the population mobility. Given that it is hard to accurately determine this time-dependent factor, it was used to dynamically adjust the model by matching the predicted cases to the registered ones. With this purpose, a control algorithm that regulates the $r_{c}$ value to match the detected infectious population in the model $\left(A_{I}+M S_{I}+S S_{I}+H_{a}+I C U+M S_{L I}\right)$ with the same kind of registered patients was implemented.

To show the effectiveness of this adjustment methodology some simulations were run and are presented in Fig. 4. To start the local virus spreading, these simulation were conducted using the arrival date of the positive imported cases registered in Argentina, temporally distributed through the fifteen age bands according to Fig. 3. Detection campaigns were considered by choosing $\delta=0.2$ and $\epsilon=0.01$. The typical SARS-CoV-2 transmission rate was taken as $\lambda=0.22$ [3].

Figure 4 shows the adjustment process result. Specifically, it presents the active cases, the cumulative recovered patients, people in ICU and the fatal cases. In all these plots solid blue, cyan and black lines represent the model predictions corresponding to the isolated (detected), not isolated (not detected) and total cases, 


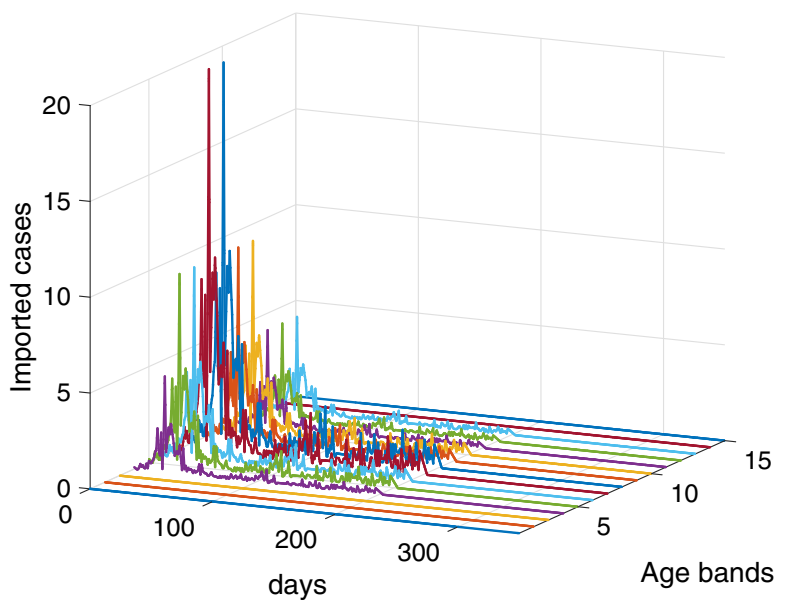

Fig. 3 Temporal profiles corresponding to the imported cases for every age band. Day 0 corresponds to $03 / 03 / 2020$

respectively. The $y$-axis values were normalised to the total population $\mathrm{N}$. On the other hand, green solid lines depicts the corresponding registered cases. The adjustment process was carried out using the isolated active cases because they are the only ones that can be contrasted against public databases. Registered active real cases were divided into two time periods. The first period was used to adjust the model (solid red line) using the proposed algorithm. The second period (solid green line) was employed to validate the previous tuning. During this last stage, the $r_{c}$ coefficient is hold constant at the last value registered during the adjustment period. More details about the tuning procedure are developed in Appendix. As it is clearly shown, Fig. 4 reveals a very good model adjustment, not only during the tuning process but also after it. This positive validation result endorses the proposed model and its possible use to predict the epidemic evolution in different scenarios, helping to qualitatively evaluate social and economic consequences of possible interventions.

\section{Simulation Results}

Several computing simulations were conducted to show the proposed dynamic model potential in predicting the effectiveness of public health policies against SARSCoV-2 pandemic. These simulations were organised in three scenarios, recreating possible pharmaceutical and non-pharmaceutical interventions. The first one only considers detection campaigns (with different degrees of intensity) and social distance or mobility restrictions as the unique possible public actions. The second and third scenarios take into account different combinations of vaccination by age bands, immunity length (on vaccinated and recovered people) and partial activity's restoring like school attendance. 

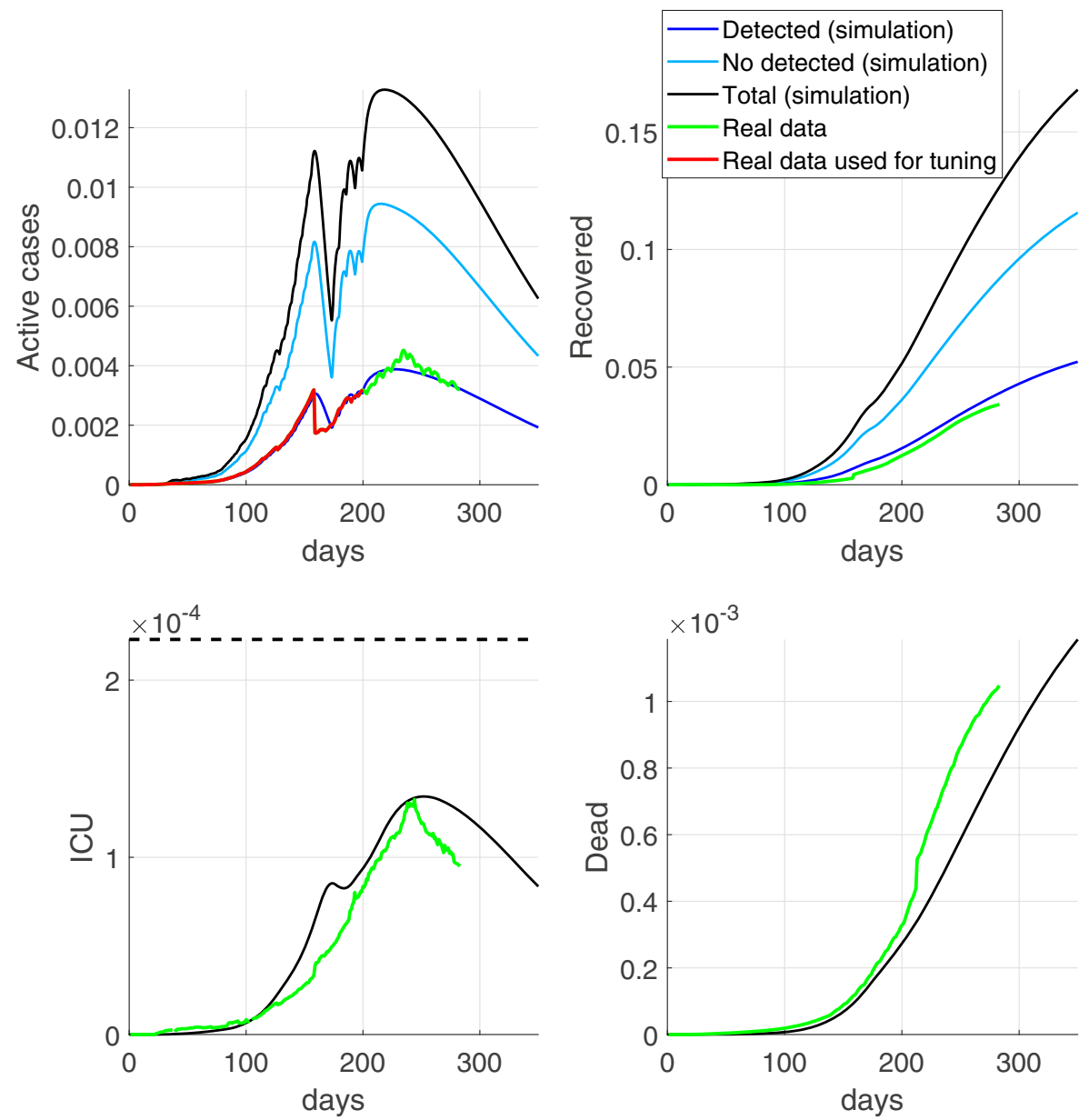

Fig. 4 Model adjustment for the Argentinian Pandemic Evolution. Solid blue: isolated (detected), cyan: not isolated (not detected) and black lines: total cases. Solid red line: real registered cases used to adjust the model. Green solid lines: real registered cases used to validate the tuning

All these scenarios were simulated considering the Argentinian context during the local SARS-CoV-2 spreading, which involves school/university attendance suspension and a sustained mobility reduction for people over 60 years old. As it was mentioned in the previous section, these situations are recreated on the risk contacts matrix reducing the values corresponding to the age bands involved in these activities. Following this criterion, the first five age bands (school to university age bands) and the last three rows and columns ( +60 years old) of the original risk contact matrix presented in Fig. 2 were multiplied by a 0.1 and a 0.4 factor, respectively. After this action, the risk contacts matrix effectively used assumes the shape shown in Fig. 5. These kinds of restrictions can be voluntary removed (partial or totally) during simulations to analyse the consequences of possible public interventions. It is important 


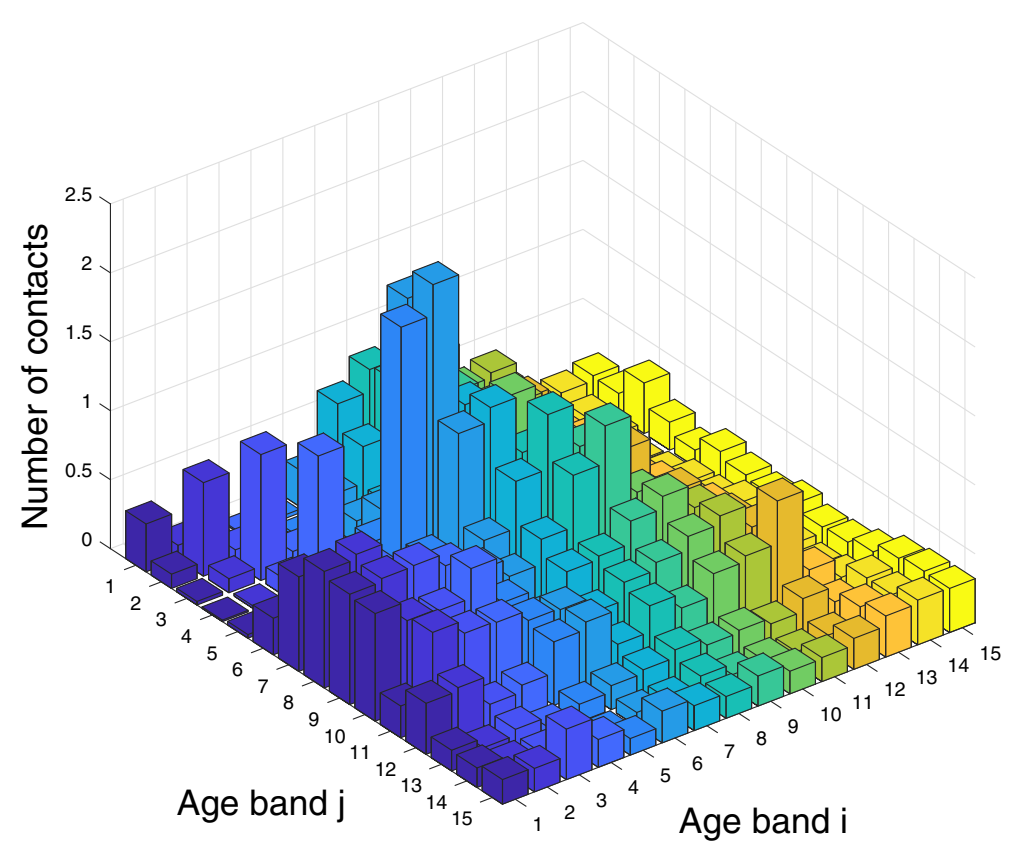

Fig. 5 Risk contacts matrix with school/university attendance suspension and mobility reduction for +60 years old people

to distinguish that general mobility restrictions, social distancing measures and similar actions are jointly represented through the $r_{c}$ coefficient and not through the risk contacts matrix manipulation.

\subsection{Scenario A: Detection Campaigns and Mobility Restrictions}

In the previously referred context, this scenario presents the predicted pandemic evolution considering detection campaigns with different intensities $(\delta)$ in combination with several social distancing/mobility restrictions levels expressed through the $r_{c}$ value. These simulations were carried out taking the parameters values previously obtained and specified for the Argentinian case and starting from an initial state where all the population is considered into the susceptible compartment. The local pandemic simulations were triggered by considering the imported cases time profiles mentioned at the end of Section 3.

Figure 6 presents the simulation results corresponding to the evolution of ICU occupancy, the cumulative number of recovered patients and the cumulative fatal cases for nine combinations of $r_{c}$ and $\delta$ values. The plots of this figure are organised in two columns considering different ICU situations. The boxes on the left column account for an unlimited ICU capacity, while the ones on the right column take into account the actual ICU saturation.

Like in the previous case $y$-axis values are normalised with respect to $\mathrm{N}$. As it can be observed, curves corresponding to different $r_{c}$ values are characterised by 

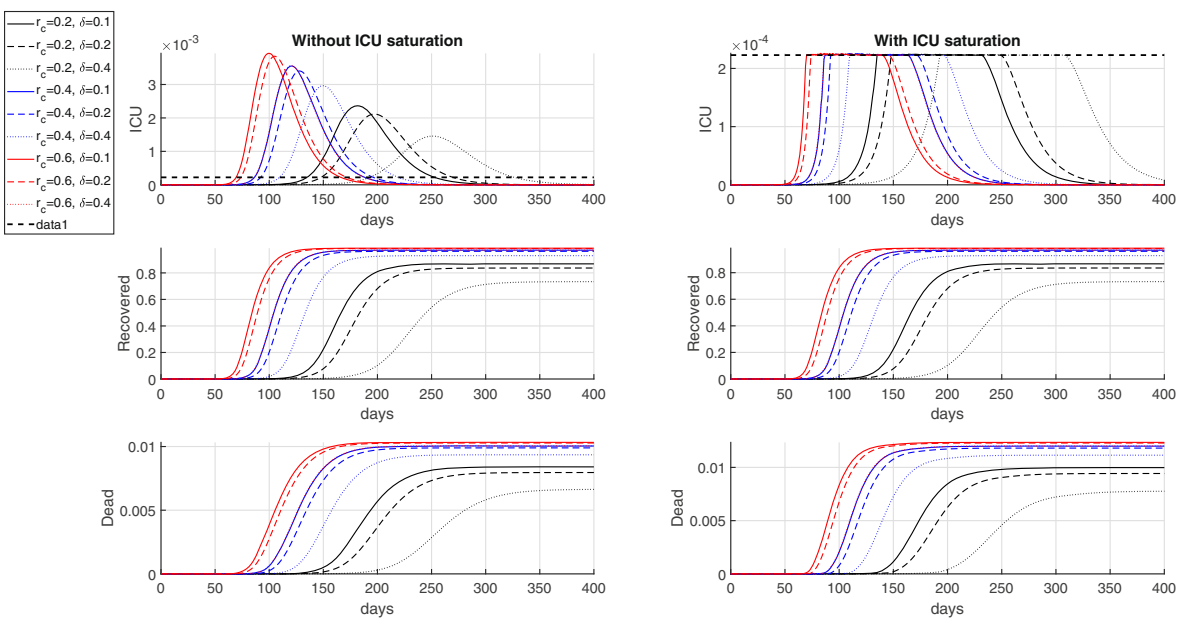

Fig. 6 Detection campaigns and mobility restrictions. Different intensity combinations. Left: without ICU saturation. Right: with ICU saturation. Black lines: $r_{c}=0.2$; blue lines: $r_{c}=0.4$ and red lines: $r_{c}=0.6$. Solid lines: $\delta=0.1$, dashed lines: $\delta=0.2$ and dotted lines: $\delta=0.4$

different colours $\left(r_{c}=0.2\right.$ in black lines; $r_{c}=0.4$ in blue lines and $r_{c}=0.6$ in red lines) and curves for different $\delta$ values are distinguished through three classes of lines ( $\delta=0.1$ in solid lines; $\delta=0.2$ in dashed lines and $\delta=0.4$ in dotted lines). Looking at this figure it is straightforward to conclude that increasing efforts in detection do not produce a substantial reduction in the final fatal or recovered cases when mobility and social distancing are not restrained in a moderate way at least. On the contrary, holding a relatively low $r_{c}$ value (reduced social mobility, social distancing, mask wearing, etc.) strengthen in a considerable way the positive effect of heavy detection campaigns on the final number of fatal cases. This behaviour can be clearly observed in the curves corresponding to $r_{c}=0.2$ (Fig. 6). These simulation results reinforce the importance of jointly manage this kind of NPIs. Analysing both figure columns it is straightforward to see that the impact of considering ICU saturation is not substantial except for the regrettable final number of fatal cases. This fact is directly related to the high probability of dying $\left(\rho_{i}\right)$ in ICU. Considering this result, all the simulations presented in the following subsections do not consider ICU saturation. The idea behind this decision is to put in evidence the hypothetical extra ICU capacity, necessary to attend to all critical patients. This information is crucial if new treatments which improve the survival rate of people in ICU arise. Also, a high ICU capacity saturation shows an undoubtedly wrong epidemic handling.

\subsection{Vaccination and Partial Activities Restoring}

The simulation scenarios presented in this subsection show the effects of different vaccination strategies combined with the reopening of specific activities like school/university attendance, mobility changes for people that belongs to the last age bands (elderly people) and a possible delayed reinfection. All these 
simulations include the period of adjustment to the Argentinian case already explained in Section 3. After this tuning process, the $r_{c}$ coefficient is hold constant up to the end of the simulations. This condition supposes no new general mobility restrictions or confinements nor substantial changes in the new social habits acquired during the pandemic time. The reopening of activities focused on specific age bands is emulated through appropriate risk contacts matrix manipulations, as it was established at the beginning of this section. A $100 \%$ vaccine effectiveness was considered. Other similar immunity percentages were tested without essential result changes and therefore are not reported here.

\subsubsection{Scenario B: Vaccination without Reinfection}

This subsection presents several vaccination strategies in combination with the school/university reopening, the end of elderly people confinement and permanent immunisation by vaccination. All the vaccination campaigns start on January 15, 2021 and last 60 days. The reopening of school/university activities and the end of mobility restrictions for elderly people is settled on February 15, 2021. These events are respectively marked with solid and dashed vertical lines in Fig. 7 where simulations results, in terms of susceptible population $(S)$, active cases, people in ICU, vaccinated people $(V)$, cumulative recovered patients $(R)$ and cumulative fatal cases $(D)$ are presented. Vertical axis values are normalised with respect to $\mathrm{N}$ with the exception of vaccinated (V). Some of these plots show in solid green line the corresponding registered cases up to $12 / 11 / 2020$. The active cases were used to adjust the model as it was previously explained. After the tuning process the $r_{c}$ coefficient is hold constant up to the end of all the simulations. In this context, solid blue lines refer
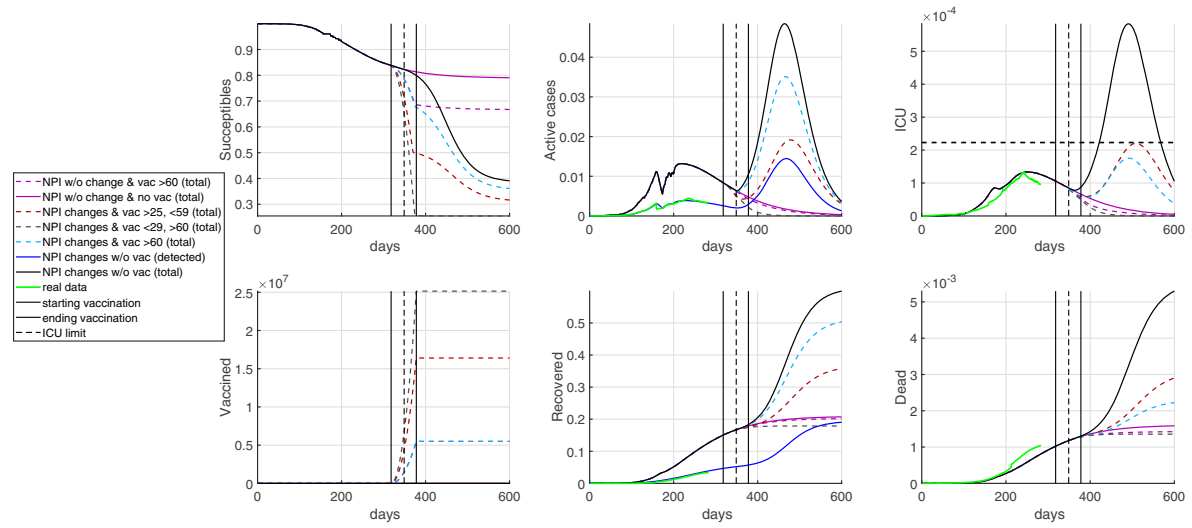

Fig. 7 Scenarios considering vaccination and activities restoring. Violet lines (solid and dashed): no changes in restrictions nor activities resumption. Violet solid lines: without new pharmaceutical interventions. Violet dashed lines with vaccination for +60 YO population. Blue, black, cyan, brown and grey lines consider the reopening of school/university activities and the end of mobility restrictions for elderly people. Solid blue lines (detected cases) and black lines (total cases) for no vaccination at all. Cyan dashed lines: vaccination for +60 YO people. Brown dashed lines: vaccination from 25 to $59 \mathrm{YO}$. Grey dashed lines: vaccination for up to 29 and over 60 YO. Green lines: real registered cases 
to the model evolution for detected cases. Lines in black represent the total cases for identical conditions. As it can be seen, this case is characterised by a heavy second wave with an unbearable number of fatal cases.

Violet lines (solid and dashed) represent the pandemic evolution when no changes in restrictions nor activities resumption takes place. The solid lines correspond to the case without new pharmaceutical interventions while dashed lines consider vaccination for over 60 years old population. As can be seen from the figure, although vaccination of this age group (without new activities) produces a somewhat faster drop of the active cases than without vaccination, the effect is not very noticeable given that these age bands do not actively participate in the distribution of the virus for being "self-isolated". Thus, the effect of vaccination on this age range does not have a remarkable effect on the pandemic extension, but it has an important collateral advantage which is the end of elderly people auto isolation and a decrease of disease mortality (since it is the range most affected age group). In this sense, it should be noted that the violet lines represent a fictional scenario because isolation measurements cannot be sustained indefinitely. It is only included for comparative issues.

The remaining-coloured dashed lines represent different vaccination alternatives, always considering the reopening of school/university activities and the end of mobility restrictions for elderly people on the dates already specified. In this context, cyan dashed lines represent the pandemic evolution when the vaccination campaign only includes over 60 years old still susceptible people (4.8M people approximately), brown dashed lines consider vaccination on still susceptible people from 25 to 59 years old (12.1M people approximately) and grey dashed lines take into account vaccination on still susceptible people up to 29 and over 60 years old (22M people approximately). It should be noted that all these last fictional vaccination campaigns only consider susceptible people to reduce the number of vaccines necessary for the first campaign. It is interesting to note that the first two vaccination strategies present an important second wave. The height of this wave is lower in the second alternative (brown lines) because of the higher number of vaccinated people. Paradoxically, as it can be observed, this last option also determines a bigger number of fatal cases and people in ICU because elderly susceptible people are not being protected. The second alternative (brown lines) reach the ICU saturation limit, whose total capacity is denoted by the horizontal dotted black line, meanwhile the first alternative (cyan lines) remains below it. Further reductions on the ICU occupancy and fatal cases figures can be reached by conditioning vaccination to sensitive population segments, i.e. with the higher values of $\sigma_{s}$ and $\mu$ on Table 4.

Finally, the third vaccination strategy (dashed grey lines) avoids the second wave at expenses of the high number of vaccines applied (22M of people approximately). This case allows a fast epidemic extinction, a reduced ICU occupancy and the lowest number of fatal cases, even below to the strategy corresponding to the dashed violet lines that consider vaccination over 60 years old people without any activities reopening.

Despite that Fig. 7 only shows the temporal evolution of the added main general variables, the model contains a lot of internal information. In this sense, Fig. 8 presents the time evolution corresponding to all the model variables by age bands. 

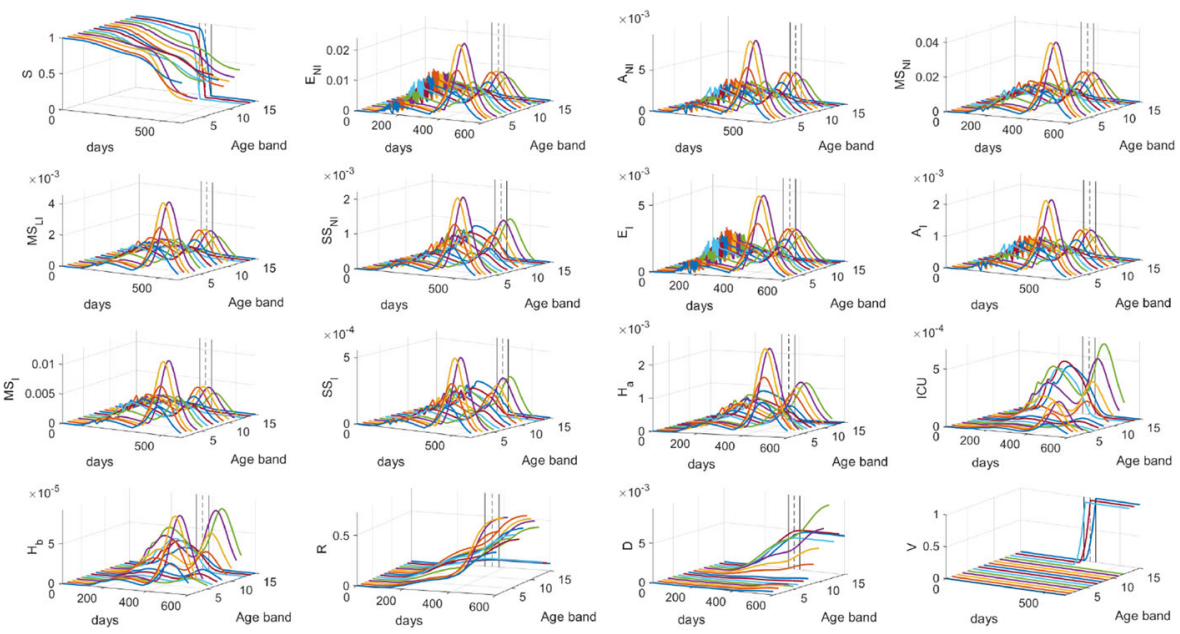

Fig. 8 Time evolution corresponding to all the model variables by age bands for vaccination over 60 years old still susceptible people with activities reopening (dashed cyan lines in Fig. 7)

The case plotted is one of the previously seen (dashed cyan line in Fig. 7) and considers vaccination over 60 years old still susceptible people with activities reopening. It is interesting to note how the vaccination period and immunised age bands can be clearly observed in the first and last plots of this figure. Besides, it should be noted that the last age bands are the most affected by fatal cases and that vaccination stops the deaths rising after the unavoidable delay originated by people already infected at the vaccination time. It is very important to see from this plot that, to significantly reduce fatality, vaccination should also include the previous age band (55 to 59 years old - green solid line). Finally, it is valuable to observe that is the population into the lower age bands (non-vaccinated) the one that dominates the virus transmission from the activities reopening.

\subsubsection{Scenario C: Vaccination with Reinfection}

Until the submission of this work there was no certainty about permanent immunisation against SARS-CoV-2. In this context, this subsection analyses some of the scenarios previously presented but considering that antibodies by cure or vaccination only provides for a temporal immunisation. Like in the previous subsection, all the simulated vaccination campaigns start on January 15, 2021, and the reopening of school/university activities and the end of mobility restrictions for elderly people is settled on February 15, 2021. These events are respectively marked with solid and dashed vertical lines in Fig. 9, where simulations results in terms of susceptible population $(S)$, active cases, people in ICU, vaccinated people $(V)$, cumulative recovered patients $(R)$ and cumulative fatal cases $(D)$ are presented. Vertical axis values are normalised with respect to $\mathrm{N}$ with the exception of vaccinated $(\mathrm{V})$. 

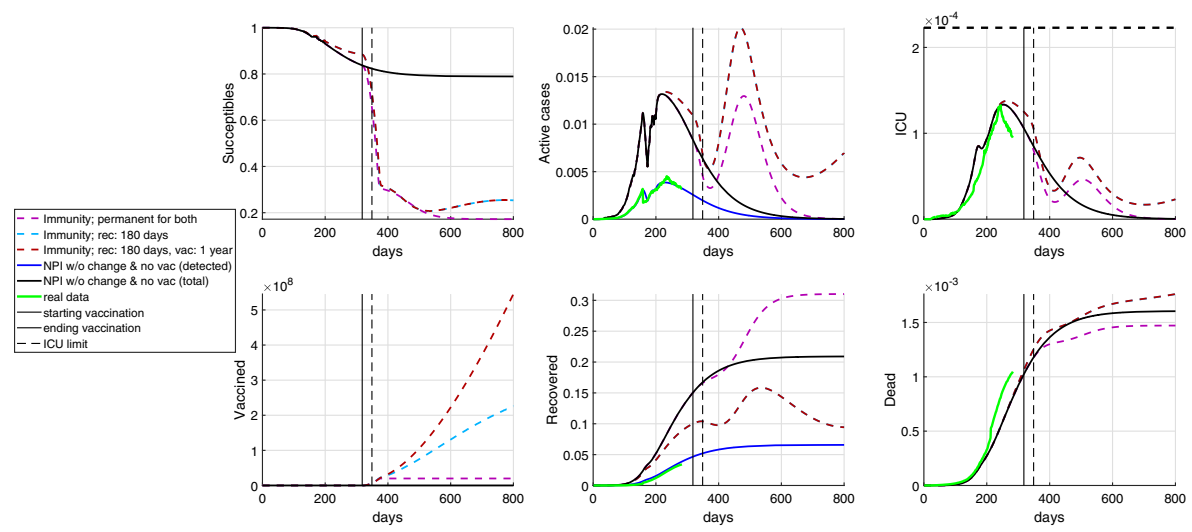

Fig. 9 Scenarios considering vaccination, activities openings and reinfection. Solid blue (detected) and black (total infected people) predicted by the model without vaccination and activities restoring. Violet dashed lines: permanent immunisation for both vaccinated and cured people. Cyan lines: consider a period of 180 days immunity length for recovered people. Brown dashed lines consider a period of 180 days immunity length for recovered people and a year of immunity length for vaccinated people. Green lines: real registered cases

Specifically, Fig. 9 depicts in solid blue and black lines the detected and total infected people respectively predicted by the model in the case of considering no vaccination nor activities restoring. Green lines denote the corresponding real registered cases. Different immunity length scenarios are presented in violet, cyan, and brown dashed lines. All of them consider vaccination over 20 years old people from the specified date. The first scenario, in violet dashed lines, supposes permanent immunisation for both vaccinated and cured people. The other cases consider a period of 180 days immunity length for recovered people (in cyan dashed lines), and the previous condition plus a year of immunity length for vaccinated people (in brown dashed lines).

As it can be observed both last cases (in cyan and brown dashed lines) present a similar evolution in most of the plots because reaching a permanent immunisation through one dose or through periodic vaccination is totally equivalent. The main difference lies in the final number of vaccines necessary to reach the immunity for population over 20 years old. The second wave observed in these two cases is produced by the activities reopening in combination with the low age bands periodic reinfection (not vaccinated). This second wave is also present in the case of permanent immunisation for cured and vaccinated people (violet dashed lines), but it is considerably lower because in this last case there is no possibility of reinfection. This is the cause of the epidemic extinction that can be observed for this case but not in the previous ones. Any of the vaccination scenarios considered in this subsection present a higher number of active patients than the case with no vaccination nor activities reopening (black solid lines), and a similar number of final fatal cases. However, despite this apparent disadvantage, the former alternatives allow to partially recover the economic activity and the general health status of the population. 


\section{Conclusions}

An age-band compartmentalised model for predicting COVID-19 spread was presented. The model was fitted to the epidemic situation reported in Argentina and different scenarios involving early detection campaigns, vaccination of age bands, reopening of activities and potential loss of acquired immunity were evaluated.

The first scenario emphasised that detection campaigns should be combined with NPIs like mobility reduction and social distancing. A second scenario involving vaccination of different age bands, school activities openings and the ending of mobility restriction for +60 years old people showed that second waves of infections may appear depending on the selected group to be vaccinated. Therefore, determining the sensitive population is important when the number of available vaccines is limited. For instance, when +60 age bands are vaccinated the fatality can be reduced with respect to other combinations that do not consider these age bands. Also, the results show that including the previous band (i.e. the 55-59 age band) in the vaccination campaign is important to reduce even more the mortality, suggesting a decedent age order for vaccination. Possible reinfection after a finite immunity period was considered in a third scenario. Although new infection waves appear after activities reopening, the vaccination avoids surpassing the healthcare system capacity and reducing the mortality rate even with respect to alternatives that do not include vaccination nor restoring of forbidden activities.

In future works, the model can be considered in a closed-loop control framework in order to determine sanitary actions that can be adapted according to the availability of new treatments, medical resources and the evolution of the disease.

\section{Appendix}

This section is devoted to extending the tuning process explanation. The model variable $r_{c}$ represents a transmission rate modulation variable that concentrates different factors like mobility restrictions, the effect of wearing masks, social distance policies, etc. (see Table 2). Given that it is hard to accurately determine this time-dependent factor, it was adjusted dynamically by matching the predicted active cases to the registered ones. With this purpose, a control algorithm that regulates the $r_{c}$ value to match the detected infectious population in the model with the same kind of registered patients was implemented. This controller is designed using the theoretical sliding mode framework [25]. It is important to note that the controller is only used to adjust the model and not as an NPI. Figure 10 depicts a schematic diagram showing the control structure used for the tuning process. Some of the model inputs correspond to parameters obtained through the real data processing like $\sigma_{s}, \mu, \rho$ while others depend on considerations like the immunity period length, the intensity of test campaigns, percentage of vaccinated people per age band and distribution of imported cases. As it is sketched in Fig. 10, the tuning loop modifies $r_{c}$ based on the comparison between the model active cases and the real active cases, denoted here as $A C_{M}=A_{I}+M S_{I}+S S_{I}+H_{a}+I C U+M S_{L I}$ and $A C_{R}$ respectively. 


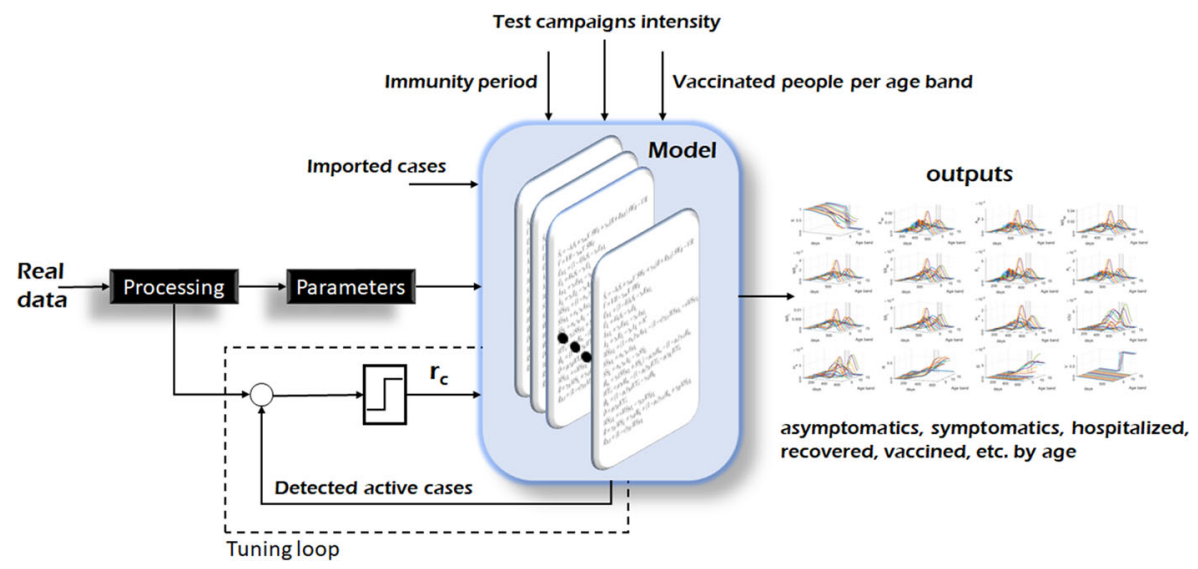

Fig. 10 Schema for the model tuning process: a controller adjusts the $r_{c}$ value to match model active cases with the registered ones

In this way, the controller tries to drive the system model to fulfil the condition $\sigma(t)=A C_{R}-A C_{M}=0$ following the time evolution of real active cases. To this end, the control action is defined as $u(t)=(\operatorname{sign}(\sigma(t))+1) / 2$, being equal to 1 (total mobility freedom) when $A C_{M} \leq A C_{R}$ and 0 (total mobility restriction) when $A C_{M}>A C_{R}$. Under the theoretical sliding mode control framework, this fast switched action will drive the system to operate with an average $r_{c}$ value matching the NPI policies applied by the government. To ensure the controller effectiveness in reaching the control objective, the design has to satisfy some theoretical issues: to comply the transversality condition and to present enough control action. These conditions were evaluated and are fulfilled by the proposed controller. For a matter of space and scope, the analysis of these conditions is not included in the paper. The interested reader is referred to [25].

In Fig. 4, the red line in the first subplot represents the time interval when this controller is active, i.e. the tuning phase. Then, the controller is disconnected, fixing $r_{c}$ to the proper averaged value of $u(t)$ for the rest of the simulation. In this way, a proper $r_{c}$ value can be estimated and used for different scenarios simulation.

Funding This work was financed by the Argentinian Government (UNLP I216, I253 and I258, CONICET PIP 0837 and 2595, PICT 2017-3211 and MINCYT BSAS28COVID FEDERAL).

\section{Declarations}

Conflict of Interest The authors have no relevant financial or non-financial interests to disclose.

\section{References}

1. Banholzer N, van Weenen E, Kratzwald B, Seeliger A, Tschernutter D, Bottrighi P, Cenedese A, Salles JP, Vach W, Feuerriegel S (2020) 
2. Carletti T, Fanelli D, Piazza F (2020) COVID-19: The unreasonable effectiveness of simple models. Chaos, Solitons \& Fractals: X 5:100034

3. Tagliazucchi E, Balenzuela P, Travizano M, Mindlin GB, Mininni PD (2020) Lessons from being challenged by COVID-19. Chaos, Solitons \& Fractals 137:109923

4. Fang Y, Nie Y, Penny M (2020) Transmission dynamics of the COVID-19 outbreak and effectiveness of government interventions: A data-driven analysis. J Med Virol 92:645-659

5. Metcalf CJ, Morris DH, Park SW (2020) Mathematical models to guide pandemic response. Science 369:368-369

6. NTJ, Bailey TJ et al (1975) The mathematical theory of infectious diseases and its applications. Charles Griffin \& Company Ltd, 5a Crendon Street, High Wycombe Bucks HP13 6LE

7. Hethcote WH (2000) The mathematics of infectious diseases. SIAM Rev 42:599-653

8. Kermack WO, McKendrick AG (1937) Contributions to the mathematical theory of epidemics IV. Analysis of experimental epidemics of the virus disease mouse ectromelia. Epidemiology \& Infection 37:172-187

9. Boatto S, Bonnet C, Cazelles B, Mazenc F (2018) SIR model with time dependent infectivity parameter: approximating the epidemic attractor and the importance of the initial phase. Available from: https://hal.inria.fr/hal-01677886

10. Huo H-F, Yang P, Xiang H (2019) Dynamics for an SIRS epidemic model with infection age and relapse on a scale-free network. J Frankl Inst 356:7411-7443

11. Liu Q, Jiang D, Hayat T, Alsaedi A (2019) Dynamics of a stochastic multigroup SIQR epidemic model with standard incidence rates. J Frankl Inst 356:2960-2993

12. Ivorra B, Ferrández MR, Vela-Pérez M, Ramos AM (2020) Mathematical modeling of the spread of the coronavirus disease 2019 (COVID-19) taking into account the undetected infections. The case of China. Commun Nonlinear Sci Numer Simul 88:105303

13. Chowdhury R, Heng K, Shawon MSR et al (2020) Dynamic interventions to control COVID-19 pandemic: a multivariate prediction modelling study comparing 16 worldwide countries. Eur J Epidemiol 35:389-399

14. Adiga A, Dubhashi D, Lewis B et al (2020) Mathematical models for COVID-19 pandemic: A comparative analysis. J Indian Inst Sci 100:793-807

15. Mohamadou Y, Halidou A, Kapen PT (2020) A review of mathematical modeling, artificial intelligence and datasets used in the study, prediction and management of COVID-19. Appl Intell 50:3913-3925

16. Cohen T, White P (2016) Transmission-dynamic models of infectious diseases. Infectious Disease Epidemiology (Oxford Specialist Handbooks) - Chapter 6 Oxford University Press

17. Dureau J, Kalogeropoulos K, Baguelin M (2013) Capturing the time-varying drivers of an epidemic using stochastic dynamical systems. Biostatistics 14(3):541-555

18. Tuite AR, Fisman DN, Greer AL (2020) Mathematical modeling of COVID-19 transmission and mitigation strategies in the population of Ontario, Canada. CMAJ 192(19):E497-E505

19. Leung K, Jit M, Lau EHY, Wu1 JT (2017) Social contact patterns relevant to the spread of respiratory infectious diseases in Hong Kong. Scientific Reports 7(1):2045-2322

20. Mossong1 J, Hens N, Jit M, Beutels P, Auranen K, Mikolajczyk R, Massari M, Salmaso S, Scalia Tomba G, Wallinga J, Heijne J, Sadkowska-Todys M, Rosinska M, Edmunds WJ (2008) Social contacts and mixing patterns relevant to the spread of infectious diseases. PLoS Med 5(3):381-391

21. Database of the National Health Ministry (Argentina). http://datos.salud.gob.ar/dataset/covid-19casos-registrados-en-la-republica-argentina. Accessed on: 12/11/2020

22. Bi Q et al (2020) Epidemiology and transmission of COVID-19 in 391 cases and 1286 of their close contacts in Shenzhen, China: a retrospective cohort study. The Lancet Infectious Diseases 20(8):911-919

23. Tindale LC, Stockdale JE, Coombe M, Garlock ES, Lau WYV, Saraswat M, Zhang L, Chen D, Wallinga J, Colijn C (2020) Evidence for transmission of COVID-19 prior to symptom onset. eLife 9:e57149

24. Wölfel R, Corman VM, Guggemos W, Seilmaier M, Zange S, Müller MA, Niemeyer D, Kelly TCJ, Vollmar P, Rothe C, Hoelscher M, Bleicker T, Brünink S, Schneider J, Ehmann R, Zwirglmaier K, Drosten C, Wendtner C (2020) Virological assessment of hospitalized cases of coronavirus disease 2019. medRxiv 2020.03.05.20030502

25. Shtessel Y, Edwards C, Fridman L, Levant A (2014) Sliding Mode Control and Observation. Springer, Berlin

Publisher's Note Springer Nature remains neutral with regard to jurisdictional claims in published maps and institutional affiliations. 